\title{
Predictive model of preoperative bacteriuria in urological surgery
}

\begin{abstract}
Introduction: In urological surgery antimicrobial prophylaxis is recommended, in order to decrease infective complications. Even more, asymptomatic bacteriuria should be ruled out before surgery, and treated, before the procedure is performed. But the urine culture gives delayed information of the bacteriuria status. So it would be important to have a good estimation of the bacteriuria status in the moment of the surgery.
\end{abstract}

Objectives: Develop a predictive model of preoperative bacteriuria, based on clinical data and urine analysis. Evaluate the accuracy of the prediction. Validate the model in a patient population different from the one used to elaborate it.

Material and methods: Both clinical and urine analysis from 700 patients were extracted for the elaboration of a predictive model of bacteriuria, using binary logistic regression. Data from 150 patients more were used for the validation of the model.

Results: The predictive model included the presence and kind of foreign body in the urine track, presence of nitrites in urine, and presence and level of leukocyte esterase in urine. The model has an AUC of the ROC curve of 0,906 , showing an excellent predictive capacity. In the validation sample the model has an AUC of 0,872 , demonstrating a very good internal validity.

Keywords: asymptomatic bacteriuria, predictive model, urine culture, urine test, urological surgery, infective complications
Volume 4 Issue 3 - 2017

\author{
Aragón Chamizo², Javier Mayor de Castro', \\ Ramírez Martín D', Herranz Amo F', Burgos \\ Revilla FJ, ${ }^{3}$ Hernández Fernández $\mathrm{C}^{\prime}$ \\ 'HGU Gregorio Marañón, Spain \\ ${ }^{2}$ HUF Jiménez Díaz, Spain \\ ${ }^{3}$ HGU Ramón y Cajal, Spain
}

Correspondence: Javier Mayor de Castro, HGU Gregorio Mara $\tilde{A} \pm \tilde{A}^{3} n, C / D r$ Esquerdo 46 PC 28007 Madrid (Madrid), Spain,Tel +34659614853, Email jmayordecastro@gmail.com

Received: April 21, 2015 | Published: March 30, 2017

\section{Introduction}

The surgery affecting the urinary track is defined as Clean Contaminated, as far as urine may be a sterile fluid, or maybe not. So, an antibiotic prophylaxis is recommended. Transurethral resection of the prostate (TURP) has the best scientific evidence, with a randomized trial that proves the benefit of antibiotic prophylaxis. ${ }^{1}$ It seems intuitive that any surgical intervention affecting contaminated urine should be at bigger risk of bacteraemia, than if it is done with sterile urine. Bacteriuria is considered a definite risk factor for complications in urology surgery. ${ }^{2-4}$ However, given the gold standard for detection of bacteriuria is the urine culture, and the results are no ready until a few days after it has been collected, is not a very useful tool for preoperative evaluation. ${ }^{5}$ Bacteriuria is typically estimated by urine analysis, and urine sediment study. Leukocyte esterase and nitrites in urine are the best estimators of bacteriuria. But the decision making based on the urine analysis is not easy, anytime it is multifactorial. Even more, the clinical information from the patient is dismissed.

\section{Objectives}

i. Develop a predictive model of preoperative bacteriuria, based on clinical data and urine analysis.

ii. Evaluate the accuracy of the prediction.

iii. Validate the model in a patient population different from the one used to elaborate it.

\section{Material and methods}

To elaborate the predictive model of bacteriuria we analyzed, in a retrospective way, 700 consecutive patients undergoing programmed surgery at our Urology Department, and 150 more for the validation of the model. Items evaluated were age, gender, type of surgery, presence and kind of foreign body in the urinary tract and data from the urinanalysis. A urine sample was collected for urinanalysis and urine culture the day before surgery. Those variables were crossed with the result of the urine culture. Data were extracted; having for the predictive model 674 valid cases, once excluded those with missing data.

The predictive model of positive uroculture was built with binary logistic regression. The dependent variable was the result of the urinary culture, "positive" or negative". The variables used for the predictive model were optimizated $\mathrm{Cp}$ Mallows criterion, in order to minimize the number of variables used, pursuing make the model more useful. The accuracy of the model was evaluated by the area under the curve (AUC) of the ROC (receiver operating characteristic) curve. An order to evaluate the internal validity we have applied the predictive model to another population of 150 consecutive patients, measuring the AUC of the ROC curve in this other population.

\section{Results}

In the univariate analysis statistically significant association was found between positive uroculture and: 
I. Foreign body $\mathrm{p}<0$ ’01

II. Nitrites in the urine $\mathrm{p}<0$ ' 01

III. Leukocyte esterase in the urine $\mathrm{p}<0$ '01

IV. Hemoglobine in the urine $\mathrm{p}<0$ ' 01

V.Proteinuria $\mathrm{p}<00^{\prime} 01$

VI. Bacteriuria $\mathrm{p}<0$ ’01

In order to make the model more operative, we decided to choose the most optimized model (which would get the maximum predictive power with the smaller number of possible variables). This optimization was made following Cp Mallows Criterion. The definitive model included the following factors:

i. Presence and kind of foreign body

ii. Presence or not of nitrites in urine

iii. Presence and grade of leukocyte esterase in urine

With binary logistic regression the obtained formula was:

$$
P(\text { Positive uroculture })=\frac{1}{-\left(-3^{\prime} 158-1^{\prime} 189 C E 1+0^{\prime} 85 C E 2-1,466 C E 3+0^{\prime} 815 C E 4+2 E L 1+2^{\prime} 364 E L 2+3^{\prime} 251 E L 3+3^{\prime} 384 E L 4+1^{\prime} 672 N\right)}
$$

This formula expresses the probability of finally getting a positive urine culture. The interpretation of the possible result, as in any diagnostic tool, will depend on the chosen cutoff to determine whether the result is positive or negative (Table 1). For example, for a cutoff point of 0,12 , the model has a sensibility of $82,3 \%$ and a specificity of $90,03 \%$. The model has an area under the COR curve of 0,906 . The same model is applied to the other 150 cases, where the AUC obtained

\begin{tabular}{|c|c|c|}
\hline Cut off & Sensibility & I Specificity \\
\hline, 0000000 & 1,000 & 1,000 \\
\hline ,0159069 & 1,000 & ,991 \\
\hline ו9ו10375, & ,917 & ,295 \\
\hline ,0593367 & ,917 & ,288 \\
\hline ,0754I83 & ,917 & ,280 \\
\hline ,0912839 & ,917 & ,278 \\
\hline, 1065320 & ,906 & ,278 \\
\hline , I198928 & ,885 & , 187 \\
\hline , I2466I0 & 885 & , 181 \\
\hline , 1463916 & ,885 & , 178 \\
\hline, 2142158 & ,823 & ,097 \\
\hline ,2781609 & ,802 & ,093 \\
\hline 3000799, & ,802 & ,086 \\
\hline ,3153524 & ,802 & ,084 \\
\hline ,3245|31 & ,729 & ,059 \\
\hline ,3393536 & ,708 &, 059 \\
\hline ,3529445 & ,583 &, 042 \\
\hline ,382849। & ,583 &, 040 \\
\hline, 4156013 &, 563 & ,031 \\
\hline ,4609572 &, 552 & ,031 \\
\hline ,5061348 &, 521 & ,031 \\
\hline
\end{tabular}
is 0,872 in the COR curve. This confirms a good diagnostic capacity of the model (Figure 1).

Table I Model of cutoff, sensibility and specificity

\begin{tabular}{|c|c|c|}
\hline, 5511301 &, 458 &, 031 \\
\hline, 6108127 &, 427 &, 027 \\
\hline, 6446085 &, 417 &, 027 \\
\hline, 6881803 &, 385 &, 026 \\
\hline, 7188101 &, 344 &, 018 \\
\hline, 7319135 &, 323 &, 018 \\
\hline, 7582420 &, 219 &, 009 \\
\hline, 7764233 &, 208 &, 009 \\
\hline, 7901162 &, 198 &, 009 \\
\hline, 8432828 &, 198 &, 005 \\
\hline, 8889805 &, 188 &, 005 \\
\hline, 8964468 &, 167 &, 005 \\
\hline, 9024977 &, 104 &, 004 \\
\hline, 9081508 &, 094 &, 004 \\
\hline, 9305441 &, 021 &, 000 \\
\hline, 9524219 &, 010 &, 000 \\
\hline 1,000000 &, 000 &, 000 \\
\hline 0 & & \\
\hline & & \\
\hline
\end{tabular}

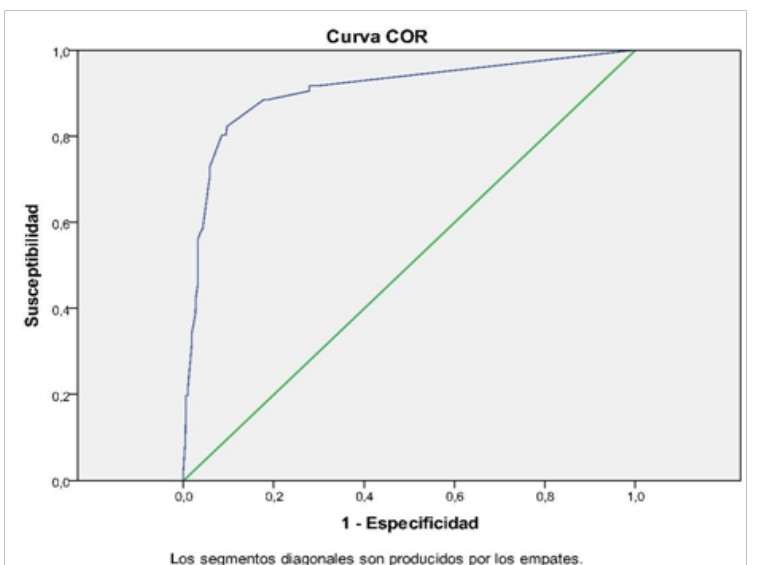

Los segmentos diagonales son producidos por los empates

Figure I COR curve. 


\section{Discussion}

The exact role of preoperative bacteriuria is about to be clarified, but is increasingly sure that it is a risk factor to develop postoperative complications. Unlike age or comorbidities, preoperative bacteriuria is a risk factor we can modify, trying to minimize its effects. 2015 EAU Guidelines assure that in procedures entering the urinary tract and breaching the mucosa, i.e. endoscopic surgeries, bacteriuria is a definite risk factor. Therefore the surgery is defined as contaminated if there is bacteriuria. Nevertheless any study in this sense is severely limited by the fact that the diagnose of bacteriuria is set by the urine culture and it takes several days to get its results. So, the decisions about antibiotic prophylaxis are taken by the type of surgery, and the condition of bacteriuria, if taken in account, is evaluated by clinical suspicion, urine dispsticks, or urinanalysis.

The recommendations we have in literature are established based in the kind of surgery and not studying the urine analyses. The best studied urological procedure is transurethral resection of the prostate: there are two large metaanalysis with prospective and randomized studies that show an important benefit of antibiotic prophylaxis with a relative risk reduction of bacteriuria of $65 \%{ }^{6-8}$ Nevertheless, in a transurethral resection of the bladder, recent recommendations advise to get antibiotic prophylaxis in function of the size of the tumor. ${ }^{9}$ In the case of ureteroscopic procedures, the factors we have to take into account are the presence of stone and his anatomic position as well as a history of urinary tract infections. ${ }^{10}$

So, probably we should adapt our antibiotic prophylaxis to the risk of having bacteriuria of our patients. Until we have a faster and more accurate diagnostic tool, the preoperative decision making should be guided through the study of the urine analyses. The application of statistical tools like binary logistic regression we propose, allows a greater diagnostic accuracy and implements new data that are not present in the basic urine analyses. The scope of the utility of this tool is limited by the complexity of the formula, and, more important, because we do not know what to do with the information we obtain. Should we not to use antibiotic prophylaxis with the patients with a very low probability of bacteriuria? Or should we give full treatment to the patients with high probability of having it? Which cut off should we use in each case? Studies using this kind of information are needed, looking forward a more patient adapted medicine.

\section{Conclusion}

It is feasible, using binary logistic regression; elaborate a reliable predictive model of preoperative bacteriuria. This model has internal validity, since its good predictive capacity has been reproduced in another sample, at the same center. The application of this model is limited by the complexity of the mathematic formula, and the lack of scientific studies that may develop intervention in function of the presence or absence of bacteriuria before the urology surgery. But it is a beginning.

\section{References}

1. Wagenlehner FM, Wagenlehner C, Schinzel S, et al. Working Group "Urological Infections" of German Society of Urology (2005) Prospective, randomized, multicentric, open, comparative study on the efficacy of a prophylactic single dose of $500 \mathrm{mg}$ levofloxacin versus $1920 \mathrm{mg}$ trimethoprim/sulfamethoxazole versus a control group in patients undergoing TUR of the prostate. Eur Urol. 47(4): 549-556.

2. El Basri A, Petrolekas A, Cariou G, et al. Clinical significance of routine urinary bacterial culture after transurethral surgery: results of a prospective multicenter study. Urology. 2012;79(3): 564-569.

3. Matsumoto M, Shigemura K, Yamamichi F, et al. Prevention of infectious complication and its risk factors after urological procedures of the upper urinary tract. Urol Int. 2012;88(1): 43-47.

4. Alapont Alacreu JM, Broseta Rico E, Pontones Moreno JL, et al. Complications of ureterorenoscopy. Actas Urol Esp. 2003;27(9): 692-699.

5. John A, Boyd JC, Lowes AJ, et al. The use of urinary dipstick tests to exclude urinary tract infection: a systematic review of the literature. $A M$ J Clin Pathol. 2006;126(3): 428-436.

6. Sohn DW, Kim SW, Hong CG, et al. Risk Factors of infectious complication after ureteroscopic procedures of the upper urinary tract. J Infect Chemother. 2013;19(6):1102-1108.

7. Berry A, Barratt A. Prophylactic antibiotic use in transurethral prostatic resection: a metaanalysis. J Urol. 2002;167(2 Pt 1): 571-577.

8. Qiang W, Jianchen W, MacDonald R, et al. Antibiotic prophylaxis for transurethral prostatic resection in men with preoperative urine containing less than 100,000 bacteria per $\mathrm{ml}$ : a systematic review. $J$ Urol. 2005;173(4): 1175-1181.

9. Alsaywid BS, Smith GH. Antibiotic prophylaxis for transurethral urological surgeries: Systematic review. Urol Ann. 2013;5(2):61-74.

10. Martov A, Gravas S, Etemadian M, et al. Postoperative Infection Rates in Patients with a Negative Baseline Urine Culture Undergoing Ureteroscopic Stone Removal: A Matched CaseControl Analysis on Antibiotic. Prophylaxis from the CROES URS Global Study. $J$ Endourol. 2015;29(2):171-180. 